This item was submitted to Loughborough's Research Repository by the author.

Items in Figshare are protected by copyright, with all rights reserved, unless otherwise indicated.

\title{
Space charge dynamics in a semiconductor superlattice affected by titled magnetic field and heating
}

\section{PLEASE CITE THE PUBLISHED VERSION}

http://dx.doi.org/10.3103/S1541308X16020035

\section{PUBLISHER}

(c) Allerton Press, Inc. Published by Springer

\section{VERSION}

AM (Accepted Manuscript)

\section{PUBLISHER STATEMENT}

This work is made available according to the conditions of the Creative Commons Attribution-NonCommercialNoDerivatives 4.0 International (CC BY-NC-ND 4.0) licence. Full details of this licence are available at: https://creativecommons.org/licenses/by-nc-nd/4.0/

\section{LICENCE}

CC BY-NC-ND 4.0

\section{REPOSITORY RECORD}

Balanov, Alexander, Alexey A. Koronovskii, Olga I. Moskalenko, Anton O. Sel'skii, and Alexander E. Hramov. 2019. "Space Charge Dynamics in a Semiconductor Superlattice Affected by Titled Magnetic Field and Heating”. figshare. https://hdl.handle.net/2134/23190. 


\title{
Изменение динамики пространственного заряда в полупроводниковой сверхрешетке под действием наклонного магнитного поля и температуры
}

(c) 2015 г. А. Г. Баланов ${ }^{1}$, А. А. Короновский ${ }^{2,3}$, О. И. Москаленко ${ }^{2,3}$, А. О. Сельский ${ }^{2,3}$,

\author{
А. Е. Храмов ${ }^{2,3}$ \\ ${ }^{1}$ Loughborough University, Department of Physics. Loughborough LE11 $3 T U$ \\ ${ }^{2}$ Саратовский государственный университет им. Н. Г. Чернышевского \\ ${ }^{3}$ Саратовский государственный технический университет им. Гагарина Ю. А.
}

E-mail: feanorberserk@gmail.com

В статье исследуется переход между режимами колебаний тока, протекающего через полупроводниковую сверхрешетку, от колебаний близких к гармоническим, вблизи начала генерации, к релаксационным. В работе показано, что тип перехода изменяется с ростом температуры. Для малых температур при переходе наблюдается бифуркация удвоения периода. С ростом температуры бифуркация удвоения периода наблюдается при все больших значениях напряжения, приложенного к сверхрешетке, до тех пор, пока переход между типами колебаний не начинает сопровождаться подавлением генерации колебаний тока, протекающего через полупроводниковую сверхрешетку.

\section{ВВЕДЕНИЕ}

Полупроводниковые сверхрешетки представляют собой структуры, состоящие из нескольких тонких слоев различных полупроводниковых материалов [1-4]. Эти полупроводниковые наноструктуры вызывают большой интерес как с точки зрения прикладной, так и фундаментальной науки [1, 5-10]. Приложенное напряжение оказывает влияние на динамику пространственного заряда в полупроводниковой сверхрешетке, вызывая генерацию колебаний тока, связанную с образованием электронных доменов [3]. В то же время, в работах [11-14] было показано, что наклонное магнитное поле оказывает большое влияние на дрейфовые скорости электронов в сверхрешетке, и, как следствие, на динамику пространственного заряда. 
Для небольших значений напряжения области повышенной концентрации пространственного заряда (электронные домены) стационарны по времени. При увеличении напряжения, приложенного к сверхрешетке, электронные домены начинают двигаться вдоль структуры. Одним из самых распространенных сценариев, приводящих к генерации колебаний тока в полупроводниковой сверхрешетке, является нормальная бифуркация Андронова-Хопфа [15]. В этом случае, при малом превышении критическим параметром (напряжением) бифуркационного значения колебания тока близки к гармоническим. С дальнейшем ростом напряжения форма колебаний тока, значительно изменяется. В настоящей работе изучены бифуркации, вызванные присутствием наклонного магнитного поля, и исследуется влияние температуры на переход между различными типами колебаний тока, протекающего через полупроводниковую сверхрешетку. Показано, что для небольших температур изменение динамики пространственного заряда осуществляется через бифуркацию удвоения периода, тогда как для более высоких значений температуры характерен срыв генерации колебаний тока, протекающего через полупроводниковую сверхрешетку.

\section{МОДЕЛЬ РАСЧЕТА ДИНАМИКИ ПРОСТРАНСТВЕННОГО ЗАРЯДА В ПОЛУПРОВОДНИКОВОЙ СВЕРХРЕШТКЕ}

Для описания коллективной динамики пространственного заряда в полупроводниковой сверхрешетке будем использовать систему самосогласованных уравнений непрерывности и Пуассона [16]

$$
\begin{gathered}
e \frac{\partial n}{\partial t}=-\frac{\partial J}{\partial x} \\
\frac{\partial F}{\partial x}=\frac{e}{\varepsilon_{0} \varepsilon_{r}}\left(n-n_{D}\right)
\end{gathered}
$$

В уравнениях (1), (2) значения концентрации электронов, плотности тока и напряженности электрического тока обозначены, соответственно, $n(x, t), J(x, t)$ и $F(x, t)$. 
Здесь $n_{D}=3 \cdot 10^{22} \mathrm{M}^{3}$ - равновесная концентрация электронов; $e>0$ - заряд электрона; $\varepsilon_{0}, \varepsilon_{r}=12.5$ абсолютная и относительная диэлектрические проницаемости.

В рамках дрейфового приближения плотность тока можно выразить следующим образом:

$$
J=e n v_{d}(\bar{F})+e D(\bar{F}) \frac{\partial n}{\partial x},
$$

где $v_{d}(\bar{F})$ - дрейфовая скорость электрона, вычисленная для среднего значения $\bar{F} ; D(\bar{F})$ коэффициент диффузии, вводимый в соответствии с [3] как

$$
D(\bar{F})=\frac{v_{d}(\bar{F}) d}{1-\exp (-e \bar{F} d / k T)} \exp (-e \bar{F} d / k T)
$$

Здесь $d=8.3$ нм - период сверхрешетки; $T$ - температура в градусах Кельвина; $k$ - постоянная Больцмана. Данный коэффициент диффузии можно не учитывать при низких температурах (порядка нескольких градусов Кельвина) и, для таких температур, в отсутствии наклонного магнитного поля дрейфовая скорость определяется формулой Эсаки-Тсу [1]. В присутствии наклонного магнитного поля для получения дрейфовых скоростей необходимо использовать численное моделирование, например так, как описано в [14]. С ростом температуры дрейфовая скорость уменьшается, тогда как наклонное магнитное поле приводит к появлению резонансных максимумов $[13,14]$, в результате чего динамика системы становится значительно сложнее. В настоящей статье использовались следующие параметры наклонного магнитного поля: индукция $B=15$ Тл, угол наклона $\theta=40^{\circ}$.

Напряжение, приложенное к полупроводниковой сверхрешетке, $V$, связано с напряженностью электрического поля как

$$
V=U+\int_{0}^{L} F d x
$$

где $U$ - падение напряжения на контактах, с учетом формирования слоев повышенной концентрации заряда вблизи эмиттера и пониженной концентрации зарядов вблизи 
коллектора сверхрешетки; $L=115.2$ нм - длина полупроводниковой сверхрешетки. Полагая, что контакты на эмиттере и коллекторе сверхрешетки омические, а плотность тока через эмиттер $J_{0}$ будет определяться проводимостью контакта $\sigma=3788 \mathrm{OM}^{-1}$ :

$$
J(0, t)=\sigma F(0, t)
$$

\section{БИФУРКАЦИЯ УДВОЕНИЯ ПЕРИОДА}

Для колебаний тока в полупроводниковых сверхрешетках типичен релаксационный характер. Однако, как уже обсуждалось выше, для значений напряжения, близких к началу генерации, вызываемой бифуркацией Андронова-Хопфа, колебания тока близки к гармоническим. Для исследования перехода от близких к гармоническим к релаксационным колебаниям будем рассматривать динамику тока и пространственно-временных доменов (областей высокой концентрации носителей заряда). Переход между различными типами колебаний тока можно продемонстрировать с помощью проекции фазовых траекторий на плоскость $(I(t-\tau), I(t))$ (где $\tau$ - время задержки, равное четверти периода колебаний), полученных с помощью метода задержек Такенса восстановления фазовых портретов по временным реализациям [17].

На рисунке 1 показаны проекции фазовых портретов и пространственно-временные зависимости концентрации носителей заряда для различных значений напряжения, приложенного к полупроводниковой сверхрешетке при температуре, близкой к абсолютному нулю $(T=4.2 \mathrm{~K})$. Для небольшого значения напряжения (близкого к началу генерации) $V=0.565 \mathrm{~B}$, форма колебаний тока близка к гармоническим и на фазовом портрете можно видеть гладкую замкнутую линию (рисунок $1, a$ ). На рисунке $1, d$ можно видеть движение доменов высокой плотности носителей заряда, которые формируют упорядоченные паттерны. Однако, увеличение напряжения до $V=0.58$ В изменяет динамику тока и пространственных доменов. Как показано на рисунке $1, b, e$, теперь предельный цикл трансформируется в цикл периода два. Из этого следует, что в системе происходит бифуркация удвоения периода. Стоит отдельно отметить, что частота колебаний при этом уменьшается вдвое. С точки зрения пространственно-временных паттернов, данная бифуркация проявляется в разделении домена вдоль координаты при прохождении отрезка от 35 до 80 нм. Можно сказать, что домен в данном случае представляет собой два максимума концентрации носителей заряда близких по высоте, так как каждому периоду 
колебаний отвечает один паттерн на пространственно-временной диаграмме. При последующем увеличении напряжения, приложенного к полупроводниковой сверхрешетке, колебания тока становятся все более отличными от гармонических и все более близкими к релаксационным (рисунок 2,c). На пространственно-временной диаграмме концентрации носителей заряда можно видеть, что один из максимумов в домене уменьшается, второй увеличивается(рисунок 2,f). В целом это приводит к уменьшению частоты следования доменов (и как следствие, к уменьшению частоты колебаний тока, о котором уже упоминалось ранее) при увеличении высоты домена (амплитуда колебаний тока также существенно увеличивается).

Рассмотрим, как влияет на динамику тока и пространственно-временных доменов вблизи бифуркации удвоения периода увеличение температуры. На рисунке 2 показаны проекции фазовых траекторий на плоскость $(I(t-\tau), I(t))$ и пространственно-временные диаграммы концентрации носителей заряда при температуре $T=100$ К. Из рисунка видно, что с увеличением температуры также наблюдается бифуркация удвоения периода. Однако, в данном случае колебания из близких к гармоническим, вблизи начала генерации (рисунок $2, a)$, становятся похожими на релаксационные (рисунок 2,b), и, только после этого, наблюдается бифуркация удвоения периода (рисунок 2,c). Более того, бифуркация наблюдается при значении напряжения, приложенного к полупроводниковой сверхрешетке, большего, чем в случае $T=4.2 \mathrm{~K}$. С точки зрения пространственно-временных паттернов носителей заряда при данной температуре задействован другой механизм перехода между режимами колебаний. В предыдущем случае, когда $T=4.2 \mathrm{~K}$, два домена объединялись в один паттерн, который изменялся до тех пор, пока один из начальных доменов не становился существенно больше другого, что приводило к удвоению периода на фазовом портрете для тока и переходу между типами колебаний. С увеличением температуры и ростом напряжения можно видеть, что электронный домен скорее расщепляется, что приводит к смене режима, после чего происходит бифуркация удвоения периода (последовательно, рисунки 2, d-f). При этом перед удвоением, цикл на фазовом портрете уже не является гладким, в отличие от случая, когда $T=4.2 \mathrm{~K}$ (см. для сравнения рисунки $1, b$ и $2, c)$.

\section{ПОДАВЛЕНИЕ ГЕНЕРАЦИИ КОЛЕБАНИЙ ТОКА}

Как следует из работы [14], с ростом температуры резонансные пики на зависимости дрейфовой скорости от напряженности электрического поля могут становиться по величине 
больше, чем пик Эсаки-Тсу. Для последующего исследования переходов между различными режимами генерации колебаний тока рассмотрим динамику проекции фазовых траекторий на плоскость $(I(t-\tau), I(t))$ и пространственно-временные диаграммы концентрации носителей заряда при изменении напряжения, приложенного к полупроводниковой сверхрешетке, при температуре $T=200$ К. Ранее упоминалось, что генерация колебаний осуществляется через бифуркацию Хопфа, колебания сразу после начала генерации близки к гармоническим. Данное поведение наблюдается для всех рассмотренных значений температуры (см. рисунки $1, a, 2, a, 3, a)$, при этом с ростом температуры значение напряжения, соответствующего началу генерации, уменьшается [14]. Однако, для температуры $T=200 \mathrm{~K}$, с ростом напряжения до значения $\mathrm{V}=0.565 \mathrm{~B}$ происходит подавление генерации колебаний тока, протекающего через полупроводниковую сверхрешетку, вызванное обратной бифуркацией Андронова-Хопфа (см. рисунок $3, b$ ). При этом, пространственное распределение носителей заряда вдоль сверхрешетки оказывается стационарным, то есть, не зависит от времени. Вдоль сверхрешетки может образовываться электронный домен, однако он неподвижен и не вызывает колебаний тока (рисунок $3, b$ ). Дальнейшее увеличение напряжения возобновляет генерацию колебаний, вновь через бифуркацию Хопфа. Бифуркации удвоения периода в этом случае не наблюдается, однако тип колебаний при этом меняется. Можно видеть, что на рисунке 3, с колебания близки к релаксационным. На пространственно-временных диаграммах концентрации носителей заряда наблюдается следующая последовательность: паттерн с единственным максимумом, стационарный паттерн, сложный паттерн с несколькими максимумами (последовательно, рисунки $3, d-f$ ).

\section{ЗАКЛЮЧЕНИЕ}

Таким образом, в работе были найдены сценарии перехода между различными типами режимов колебаний тока в полупроводниковых сверхрешетках в присутствии наклонного магнитного поля, от близких к гармоническим (вблизи начала генерации) до релаксационных. Генерация колебаний тока, связанная с движением электронных доменов вдоль структуры, реализуется как в присутствии, так и в отсутствии магнитного поля. Однако, в присутствии наклонного магнитного поля возможна бифуркация удвоения периода, сопровождаемая изменением перехода между режимами генерации колебаний тока. Можно сделать вывод, что изменение перехода между режимами колебаний связано с изменением соотношения величин пика Эсаки-Тсу и блох-циклотронных пиков на зависимости дрейфовой скорости от напряженности электрического поля, наблюдаемого при 
увеличении температуры. При небольших температурах, Т <100 К, переход осуществляется через бифуркацию удвоения периода, тогда как при более высоких температурах, $T=200$ К, бифуркация удвоения периода не наблюдается и в данном случае переход осуществляется через подавление генерации, ассоциирующееся с обратной бифуркацией Андронова-Хопфа.

Работа выполнена при поддержке Российского научного фонда, проект 14-12-00224. Научная деятельность А.О. Сельского поддержана также стипендией фонда некоммерческих программ "Династия". 


\section{СПИСОК ЛИТЕРАТУРЫ}

1. Esaki L., Tsu R. Superlattice and negative differential conductivity in semiconductors // IBM J. Res. Develop. 1970. V. 14. P. 61.

2. Shik A. Y. Superlattices-periodic semiconductor structures // Sov. Phys. Semicond. 1975. V. 8. P. 1195.

3. Wacker A. Semiconductor superlattices: a model system for nonlinear transport // Phys. Rep. 2002. V. 357. P. 1.

4. Tsu R. Superlattices to Nanoelectronics. Elsevier, 2005.

5. Romanov Y. Nonlinear effects in periodic semiconductor structures // Optika i Spektroskopiya. 1972. V. 33. P. 917.

6. Ignatov A. A., Shashkin V. I. Bloch oscillations of electrons and instability of spacecharge waves in superconductor superlattices // Sov. Phys. JETP. 1987. V. 66. № 3. P. 526.

7. Holthaus M. Collapse of minibands in far-infrared irradiated superlattices // Physical Review Letters. 1992. V. 69. P. 351.

8. Zhang Y., Kastrup J., Klann R., Ploog K. H., Grahn H. T. Synchronization and chaos induced by resonant tunneling in GaAs/AlAs superlattices // Physical Review Letters. 1996. V. 77. P. 3001.

9. Hyart T., Alexeeva J., Mattas N. V., Alekseev K. N. Terahertz bloch oscillator with a modulated bias // Physical Review Letters. 2009. V. 102. P. 140405.

10. Hramov A. E., Koronovskii A. A., Kurkin S. A., Makarov V. V., Gaifullin M. B., Alekseev K. N., Alexeeva N., Greenaway M. T., Fromhold T. M., Patane A., Kusmartsev F. V., Maksimenko V. A., Moskalenko O. I., Balanov A. G. Subterahertz chaos generation by coupling a superlattice to a linear resonator // Physical Review Letters. 2014. V. 112. P. 116603.

11. Fromhold T. M., Krokhin A. A., Tench C. R., Bujkiewicz S., Wilkinson P. B., Sheard F. W., Eaves L. Effects of stochastic webs on chaotic electron transport in semiconductor superlattices // Physical Review Letters. 2001. V. 87. № 4. P. 046803.

12. Fromhold T. M., Patanè A., Bujkiewicz S., Wilkinson P. B., Fowler D., Sherwood D., Stapleton S. P., Krokhin A. A., Eaves L., Henini M., Sankeshwar N. S., Sheard F. W. Chaotic electron diffusion through stochastic webs enhances current flow in superlattices // Nature. 2004. V. 428. P. 726.

13. Balanov A. G., Fowler D., Patane A., Eaves L., Fromhold T. M. Bifurcations and 
chaos in semiconductor superlattices with a tilted magnetic field // Physical Review E. 2008. V. 77. № 2. Р. 026209.

14. Selskii A.O., Koronovskii A.A., Hramov A.E., Moskalenko O.I., Alekseev K.N., Greenaway M.T., Wang F., Fromhold T.M., Shorokhov A.V., Khvastunov N.N., Balanov A.G. Effect of temperature on resonant electron transport through stochastic conduction channels in superlattices // Phys. Rev. B. 2011. V. 84. P. 235311.

15. Hizanidis J., Balanov A. G., Amann A., Scholl E. Noise-induced front motion: Signature of a global bifurcation // Physical Review Letters. 2006. V. 96. № 24. P. 244104.

16. Greenaway M. T., Balanov A. G., Schöll E., Fromhold T. M. Controlling and enhancing terahertz collective electron dynamics in superlattices by chaos-assisted miniband transport // Phys. Rev B. 2009. V. 80. P. 205318.

17. Takens F. Detecting strange attractors in dynamical systems and turbulence, Lectures Notes in Mathematics. N. Y.: Springler-Verlag, 1981. 
А. Г. Баланов, А. А. Короновский, О. И. Москаленко, А. О. Сельский, А. Е. Храмов Изменение динамики пространственного заряда в полупроводниковой сверхрешетке под действием наклонного магнитного поля и температуры

Рис. 1. $a, b, c$ - Фазовые портреты, восстановленные по методу Такенса (время задержки, $\tau$, составляет четверть периода колебаний). $d, e, f$ - Пространственно-временные диаграммы концентрации носителей заряда. Для температуры $T=4.2 \mathrm{~K}$, наклонное магнитное поле $B=15$ Тл, $\theta=40^{\circ} . a, d$ - для значения напряжения, приложенного к полупроводниковой сверхрешетке, $V=0.565$ В. $b, e-V=0.58$ В. $c, f-V=0.6$ В.

Рис. 2. $a, b, c$ - Фазовые портреты, восстановленные по методу Такенса (время задержки, $\tau$, составляет четверть периода колебаний). $d, e, f$ - Пространственно-временные диаграммы концентрации носителей заряда. Для температуры $T=100 \mathrm{~K}$, наклонное магнитное поле $B=15$ Тл, $\theta=40^{\circ} . a, d$ - для значения напряжения, приложенного к полупроводниковой сверхрешетке, $V=0.515 \mathrm{~B} . b, e-V=0.615 \mathrm{~B} . c, f-V=0.635 \mathrm{~B}$.

Рис. 3. $a, b, c$ - Фазовые портреты, восстановленные по методу Такенса (время задержки, $\tau$, составляет четверть периода колебаний). $d, e, f$ - Пространственно-временные диаграммы концентрации носителей заряда. Для температуры $T=200 \mathrm{~K}$, наклонное магнитное поле $B=15$ Тл, $\theta=40^{\circ} . a, d$ - для значения напряжения, приложенного к полупроводниковой сверхрешетке, $V=0.485$ В. $b, e-V=0.565$ В. $c, f-V=0.7$ В. 
Рисунок 1. А.Г. Баланов, А.А. Короновский, О.И. Москаленко, А.О. Сельский, А.Е. Храмов
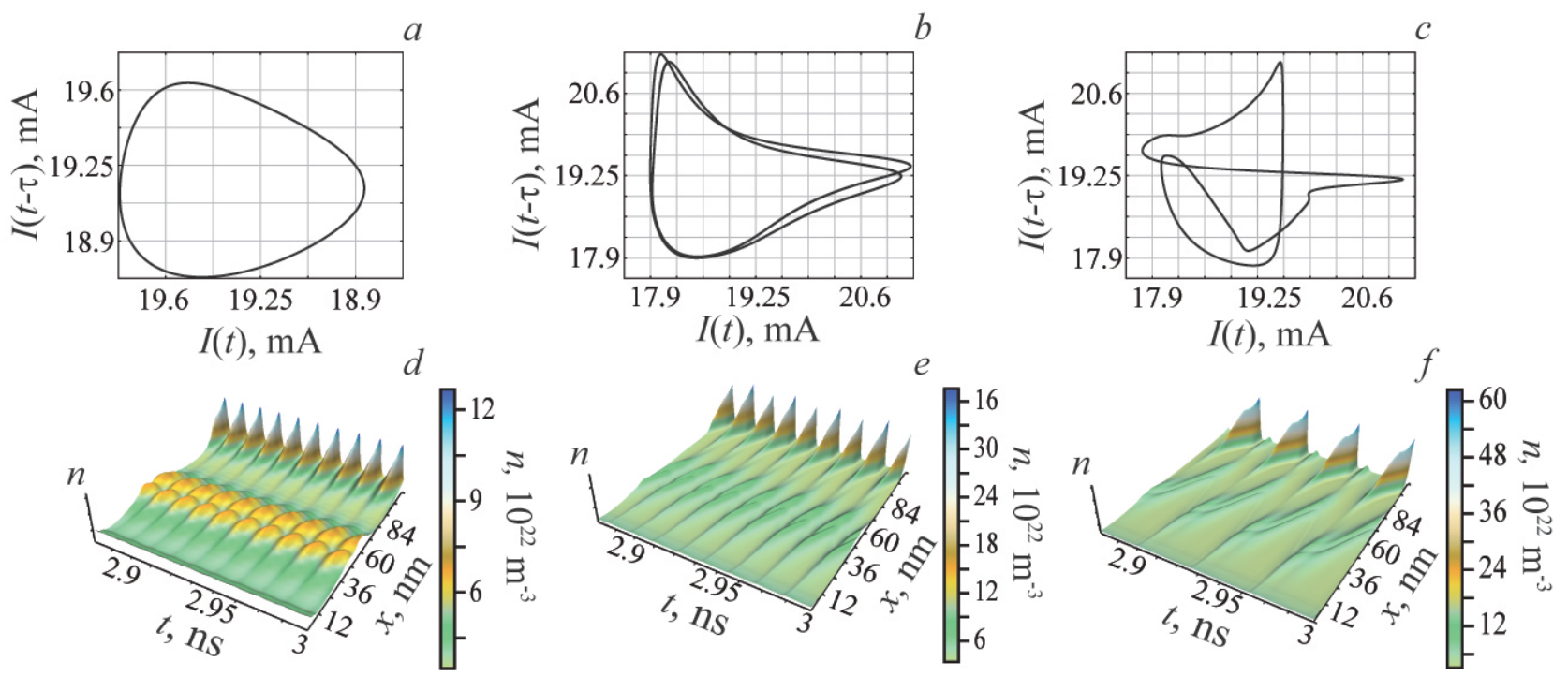
Рисунок 2. А.Г. Баланов, А.А. Короновский, О.И. Москаленко, А.О. Сельский, А.Е. Храмов
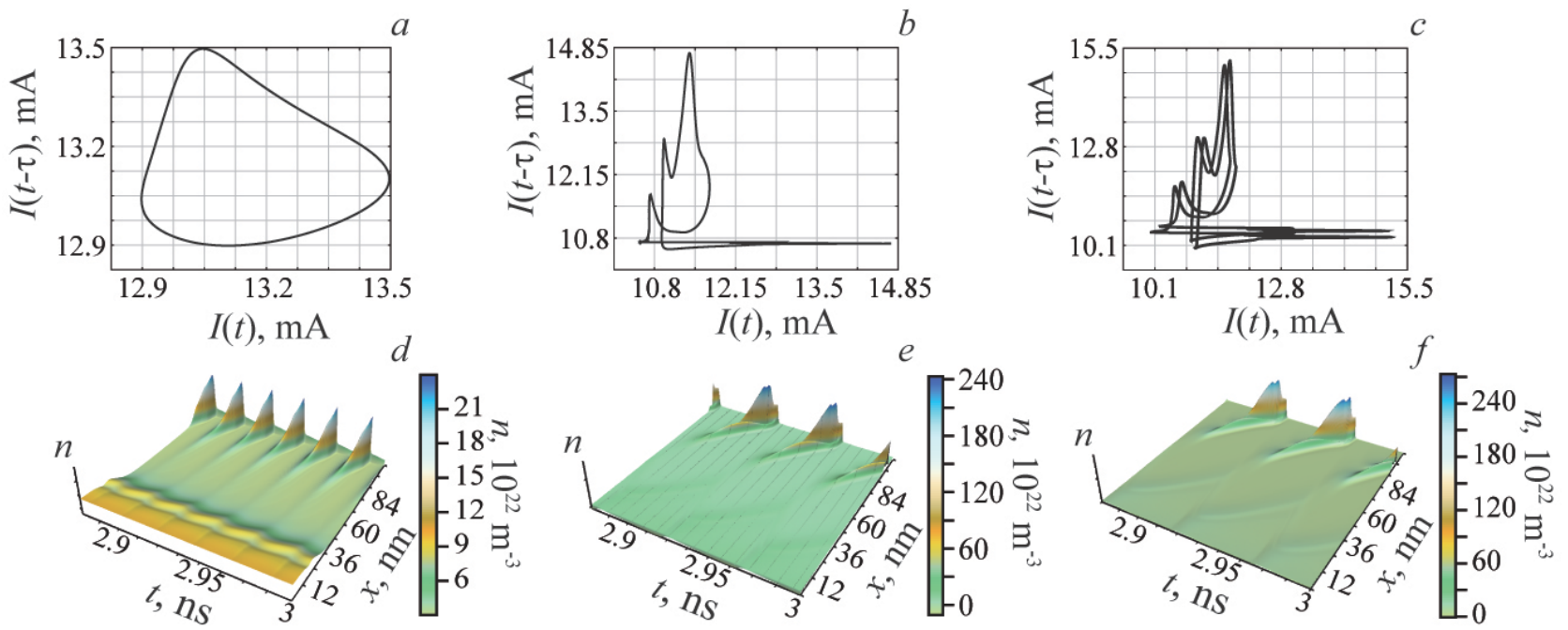
Рисунок 3. А.Г. Баланов, А.А. Короновский, О.И. Москаленко, А.О. Сельский, А.Е. Храмов
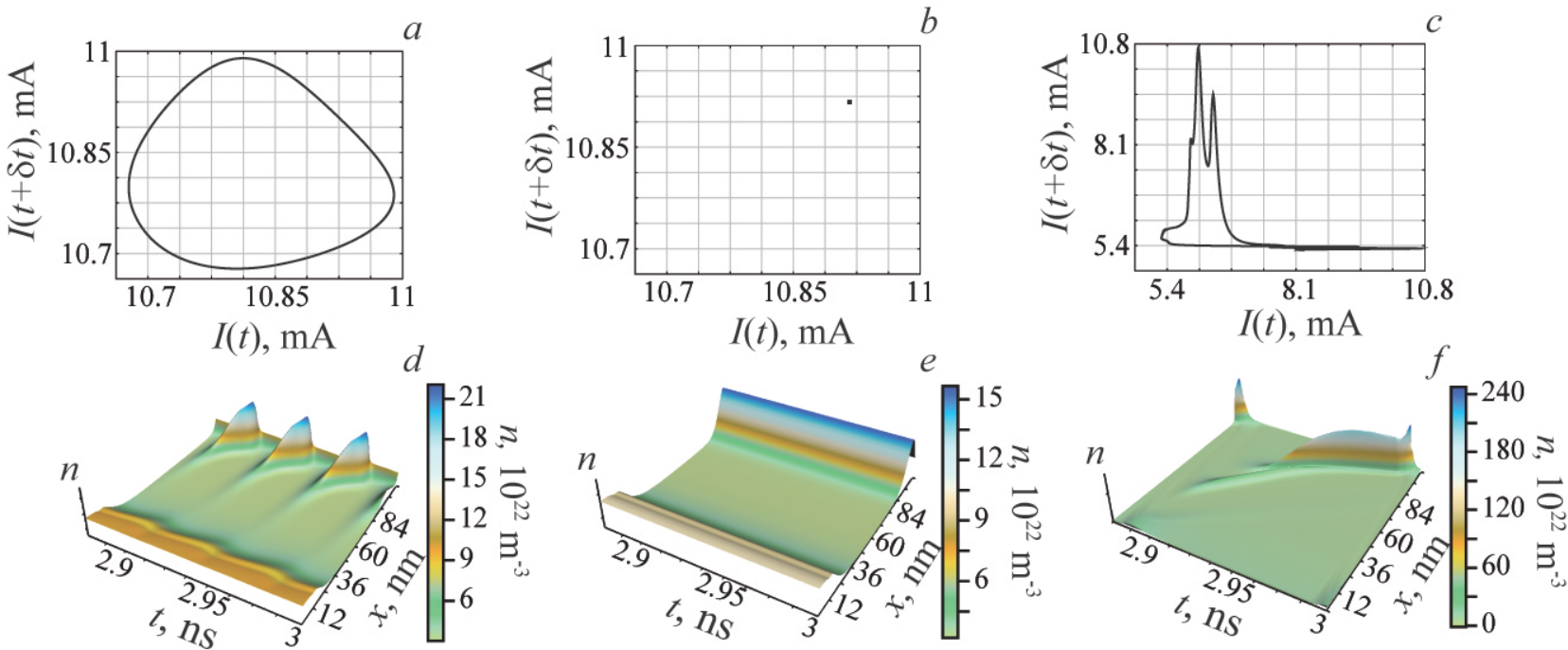


\section{ДАННЫЕ ОБ АВТОРАХ СТАТЬИ:}

А.Г. Баланов, А.А. Короновский, О.И. Москаленко, А.О. Сельский, А.Е. Храмов Изменение динамики пространственного заряда в полупроводниковой сверхрешетке под действием наклонного магнитного поля и температуры

\section{Баланов Александр Геннадьевич}

Lecturer in Physics, Loughborough University

Loughborough LE11 3TU

Тел. +44 (0) 150922 7112, e-mail: a.balanov@lboro.ac.uk.

\section{Короновский Алексей Александрович}

Профессор Саратовского Государственного Университета им. Н.Г. Чернышевского, Professor of Saratov State University

410012, Россия, г. Саратов, ул. Астраханская, д. 83

Saratov Astrakhanskaya 83 410012, Russia

Тел. .: (8452) 5145 40, e-mail: alexey.koronovskii@gmail.com

\section{Москаленко Ольга Игоревна}

Доцент Саратовского Государственного Университета им. Н.Г. Чернышевского Associate professor of Saratov State University

410012, Россия, г. Саратов, ул. Астраханская, д. 83

Saratov Astrakhanskaya 83 410012, Russia

Тел. (8452) 5145 40, e-mail: o.i.moskalenko@gmail.com

\section{Сельский Антон Олегович}

Инженер Саратовского Государственного Университета им. Н.Г. Чернышевского Engineer of Saratov State University 
410012, Россия, г. Саратов, ул. Астраханская, д. 83

Saratov Astrakhanskaya 83 410012, Russia

Тел. (8452) 5145 40, e-mail: feanorberserk@gmail.com.

\section{Храмов Александр Евгеньевич}

Профессор Саратовского Государственного Университета им. Н.Г. Чернышевского Professor of Saratov State University

410012, Россия, г. Саратов, ул. Астраханская, д. 83

Saratov Astrakhanskaya 83 410012, Russia

Тел. (8452) 5145 40, e-mail: hramovae@gmail.com 
Dynamics of the space charge in semiconductor superlattice under the influence of the titled magnetic field

\author{
(C) 2015 г. A. G. Balanov ${ }^{1}$, A. A. Koronovskii ${ }^{2,3}$, O. I. Moskalenko ${ }^{2,3}$, A. O. Selskii ${ }^{2,3, a}$, \\ A. E. Hramov ${ }^{2,3}$ \\ ${ }^{1}$ Loughborough University, Department of Physics Loughborough LE11 3TU \\ ${ }^{2}$ Department of Open Systems Physics, Faculty of Nonlinear Processes, Saratov State University. \\ Saratov 410012, Russia \\ 3 1Department of geoecology and geological engineering, Faculty of Ecology and Service, Yuri \\ Gagarin State Technical University of Saratov. Saratov 410054, Russia \\ E-mail: feanorberserk@gmail.com
}

In the present paper the transition between the different regimes of the current oscillations in the semiconductor superlattice from the oscillations like harmonic, for the start of the generation, to the relaxation oscillations is study. This transition changed with the growth of the temperature. The period-doubling bifurcation is observed for the small temperatures. The bifurcation is observed for the larger value of the voltage until the transition between the different regimes of the current oscillations do not to be followed by the stoppage of the oscillations. 